\title{
Double tract reconstruction reduces reflux esophagitis and improves quality of life after radical proximal gastrectomy for patients with upper gastric or esophagogastric adenocarcinoma
}

\section{Xin Ji}

Peking University Cancer Hospital \& Institute

\section{Cheng-Gen Jin}

Peking University Cancer Hospital \& Institute

Ke Ji

Peking University Cancer Hospital \& Institute

\section{Ji Zhang}

Peking University Cancer Hospital \& Institute

Xiao-Jiang Wu

Peking University Cancer Hospital \& Institute

Xiang-Long Zong

Peking University Cancer Hospital \& Institute

Zi-Yu Jia

Peking University Cancer Hospital \& Institute

\section{Tao Fu}

Peking University Cancer Hospital \& Institute

\section{Biao Fan}

Peking University Cancer Hospital \& Institute

\section{An-Qiang Wang}

Peking University Cancer Hospital \& Institute

\section{Zhao-De Bu}

Peking University Cancer Hospital \& Institute Jiafu Ji ( $\nabla$ jijiafu@hsc.pku.edu.cn )

Peking University Cancer Hospital \& Institute https://orcid.org/0000-0002-4235-4507

\section{Research article}

Keywords: double tract reconstruction, esophagogastrostomy, proximal gastrectomy, reflux esophagitis, quality of life 
Posted Date: August 18th, 2020

DOl: https://doi.org/10.21203/rs.3.rs-48483/v1

License: (c) (i) This work is licensed under a Creative Commons Attribution 4.0 International License. Read Full License 


\section{Abstract}

Background: The aim of the present study was to compare the difference between double tract reconstruction and esophagogastrostomy after proximal gastrectomy.

Methods: Patients who underwent radical proximal gastrectomy with esophagogastrostomy or double tract reconstruction from December 2014 to May 2019 were included in this study.

Results: Sixty-four patients were included in this study and divided into two groups according to reconstruction method. The two groups were well balanced in terms of length of postoperative hospital stay, blood loss volume, postoperative complications, and 5-year disease-free survival (DFS). The operation duration in the double tract reconstruction group was longer than that in the esophagogastrostomy group ( $240 \mathrm{~min}$ vs $195 \mathrm{~min}, P=0.001$ ). The rates of postoperative reflux esophagitis in the double tract reconstruction group and esophagogastrostomy group were $8.0 \%$ and $30.8 \%$, respectively $(P=0.032)$. The multivariable logistic regression analysis showed that the reconstruction method was the only independent risk factor for reflux esophagitis $(P=0.004)$. Patients in the double tract reconstruction group had better global health status $(P<0.001)$ and emotional functioning $(P<0.001)$, and complained less about nausea and vomiting $(P<0.001)$, pain $(P=0.039)$, insomnia $(P=0.003)$, and appetite loss $(P<0.001)$ based on the European Organization for Research and Treatment of Cancer (EORTC) QLQ-C30 questionnaire. Regarding the EORTC QLQ-STO22 questionnaire, patients in the double tract reconstruction group complained less about dysphagia $(P=0.030)$, pain $(P=$ $0.008)$, reflux $(P<0.001)$, eating $(P<0.001)$, anxiety $(P<0.001)$, dry mouth $(P=0.007)$, and taste $(P=$ 0.001). The multiple linear regression analysis showed that reconstruction method, postoperative complications, reflux esophagitis, and operation duration had a linear relationship with the global health status score.

Conclusions: Double tract reconstruction after proximal gastrectomy was comparable with esophagogastrostomy in terms of perioperative safety and 5-year DFS. Double tract reconstruction could better prevent reflux esophagitis and improve quality of life. There was a linear relationship between global health status score and reconstruction method, reflux esophagitis, postoperative complications, and operation duration.

\section{Background}

Gastric cancer is a severe threat worldwide, especially in the eastern Asian area. It has been reported that gastric cancer is the fifth most common cancer and the third leading cause of cancer-related death globally [1]. In China, gastric cancer is the third leading cause of cancer-related death, and its incidence ranks second among all malignancies. There are more than 410,000 new cases and 290,000 cancerrelated deaths each year $[2,3]$.

In recent years, the incidence of proximal gastric cancer has steadily increased [4]. With the advancements in diagnostic techniques, an increasing number of patients with early gastric cancer are 
being diagnosed. According to the Japanese Gastric Cancer Treatment Guidelines, proximal gastrectomy is recommended for patients with upper gastric adenocarcinoma at an early stage or those with adenocarcinoma of the esophagogastric junction with a tumor size less than $4 \mathrm{~cm}$ [5]. As a result, the number of patients needing proximal gastrectomy has increased. Moreover, perioperative safety and postoperative quality of life after proximal gastrectomy have gained increasing attention [6-8]. At present, a major problem of proximal gastrectomy is the high incidence of reflux esophagitis. Although partial function of the stomach is preserved, quality of life is impaired. Therefore, several reconstruction methods have been attempted to solve this problem. According to the Japanese Gastric Cancer Treatment Guidelines, double tract reconstruction and esophagogastrostomy are two of the recommended reconstructions for proximal gastrectomy [5]. Esophagogastrostomy is the traditional and most widely performed reconstruction method after proximal gastrectomy [9]. The main deficiency of esophagogastrostomy might be the high incidence of reflux esophagitis. It has been reported that the incidence of reflux esophagitis after esophagogastrostomy ranges from 9.1-35.3\% [10,11]. Conversely, some studies have reported that double tract reconstruction might reduce the incidence of reflux esophagitis after proximal gastrectomy $[12,13]$. However, double tract reconstruction is more complicated than esophagogastrostomy, and there is a lack of high-level evidence to support the advantages of double tract reconstruction [4].

Therefore, the best reconstruction method after proximal gastrectomy is still controversial. Moreover, postoperative quality of life after proximal gastrectomy with esophagogastrostomy or double tract reconstruction is rarely investigated or reported. Thus, the purpose of the present study is to compare the differences between double tract reconstruction and esophagogastrostomy after proximal gastrectomy, and to identify the superiority of double tract reconstruction.

\section{Methods}

\section{Patients}

The patients' clinical and pathological data were retrospectively collected from a prospectively maintained database at the Peking University Cancer Hospital. Between December 2014 and May 2019, patients who underwent radical proximal gastrectomy with esophagogastrostomy, or double tract reconstruction were included in the present study. All patients were diagnosed with upper-third gastric adenocarcinoma at an early clinical stage or adenocarcinoma of the esophagogastric junction with a tumor size less than $4 \mathrm{~cm}$. The diagnosis and clinical stage were confirmed by endoscopic biopsy analysis, ultrasound endoscopy, chest X-ray, abdominopelvic computed tomography scans, and laparoscopic exploration. The terminology was defined based on the Japanese classification of gastric carcinoma [14]. The clinical and pathological stages were classified based on the 8th edition Union for International Cancer Control (UICC)/American Joint Committee on Cancer (AJCC) TNM staging system [15]. 
This study was performed with approval from the Ethics Committee of Peking University Cancer Hospital (reference no. 2018KT97). Informed consent was obtained from each enrolled patient.

\section{Surgical procedure}

The surgical procedure in our department was performed in accordance with the Japanese Gastric Cancer Treatment Guidelines [16]. Radical proximal gastrectomy included resection of the proximal stomach and part of the abdominal esophagus and preserved more than half of the stomach. The reconstruction method was discussed and determined by both the patients and surgeons before surgery. The degree of lymph node dissection (LND) was also performed in accordance with the Japanese treatment guidelines [16]. D1 or D1 + lymphadenectomy was chosen according to the disease stage. Esophagogastrostomy reconstruction was performed by an end-to-side anastomosis between the esophagus and remnant stomach. The anastomosis was located on the anterior wall of the remnant stomach. For double tract reconstruction, the jejunum $25 \mathrm{~cm}$ distal to the Treitz ligament was transected, distal limb of the jejunum was lifted to prepare the esophagojejunostomy. An end-to-side esophagojejunostomy was performed with a circular stapler, and the jejunal stump was closed with a linear stapler. Next, a side-to-side gastrojejunostomy was performed $15 \mathrm{~cm}$ below the esophagojejunostomy. Finally, a side-to-side jejunojejunostomy was performed $15-20 \mathrm{~cm}$ below the gastrojejunostomy.

\section{Clinical and pathological characteristics}

The clinical and pathological characteristics of patients collected from the database included age, gender, body mass index (BMI), tumor location, proximal and distal margins, degree of differentiation, Lauren classification, tumor size, pT stage, pN stage, pTNM stage, number of harvested lymph nodes (LNs), degree of LND, lymphovascular invasion (LVI), perineural invasion (PNI), and adjuvant chemotherapy. The intraoperative and postoperative parameters included postoperative hospital stay, blood loss volume, operation duration, postoperative complications, 30-day reoperation rate, and 30-day mortality rate. Complications were defined according to the Clavien-Dindo classification system [17].

\section{Follow-up}

All patients were recommended to go to the outpatient clinic to receive re-examinations every three months in the first three years after the operation, every six months in the next two years and every year thereafter. The re-examinations mainly included physical examination, laboratory blood tests, and computed tomography scans. Endoscopy was recommended once a year after the operation. The Los Angeles classification was applied to evaluate the degree of reflux esophagitis [18]. Patient quality of life was evaluated by the validated Chinese Mandarin edition of the EORTC QLQ-C30 version 3.0 questionnaire and EORTC QLQ-STO22 questionnaire. Permission to use these questionnaires in the 
present study was obtained from the EORTC Quality of Life Group. The patients were invited to complete these two questionnaires approximately twelve months after the operation. The last follow-up date was May 2020. The follow-up assessments were conducted mainly through telephone interviews, email communication, or outpatient examinations.

\section{Statistical analysis}

All statistical analyses were performed using IBM SPSS Statistics 26.0 software (SPSS Inc., Armonk, NY). Continuous variables with normal or skewed distributions are expressed as the mean \pm standard deviation or median with interquartile range, respectively. Student's t-test or Mann-Whitney U test was used to compare variables with normal or skewed distributions among groups, respectively. Categorical variables are represented by the number of cases with percentage and were compared by the chi-square test or Fisher's exact test. Kaplan-Meier survival analysis and the log-rank test were used to compare DFS between the two groups. Univariate analysis and multivariate logistic regression analysis were conducted to identify the risk factors for reflux esophagitis. Simple and multiple linear regression analyses were applied to identify factors that might affect the global health status score in the EORTC QLQ-C30 questionnaire. A linear regression equation was established to describe the factors related to global health status. $P<0.05$ (two-sided) was considered significant in the statistical analysis.

\section{Results}

\section{Clinical and pathological characteristics}

Altogether, sixty-four patients were included in this study. Thirty-nine patients underwent esophagogastrostomy reconstruction and twenty-five patients underwent double tract reconstruction. Age, gender, BMI, tumor location, proximal and distal margins, differentiation, Lauren classification, number of harvested LNs, and LVI were comparable between the two groups (Table 1). The proportion of patients with early-stage disease was higher in the double tract reconstruction group than in the esophagogastrostomy group $(P=0.031)$. The tumor size was smaller in the double tract reconstruction group than in the esophagogastrostomy group $(1.5 \mathrm{~cm}$ vs. $3.0 \mathrm{~cm}, P=0.010)$. The proportion of $\mathrm{D} 1+$ lymphadenectomy was lower in the double tract reconstruction group than in the esophagogastrostomy group (56.0\% vs. $79.5 \%, P=0.045)$. The rate of $\mathrm{PNI}$ was lower in the double tract reconstruction group than in the esophagogastrostomy group $(12.0 \%$ vs. $38.5 \%, P=0.044)$. Fewer patients received adjuvant chemotherapy in the double tract reconstruction group than in the esophagogastrostomy group (32.0\% vs. $59.0 \%, P=0.035)$. 
Table 1

Clinical and pathological characteristics of the patients

\begin{tabular}{|c|c|c|c|}
\hline Clinicopathological characteristics & $E G(n=39)$ & DT $(n=25)$ & $P$ value \\
\hline Age, mean $\pm S D$, year & $62.1 \pm 9.1$ & $64.5 \pm 8.9$ & 0.314 \\
\hline Gender, n (\%) & & & 0.137 \\
\hline Male & $31(79.5 \%)$ & $24(96.0 \%)$ & \\
\hline Female & $8(20.5 \%)$ & $1(4.0 \%)$ & \\
\hline $\mathrm{BMI}$, mean $\pm \mathrm{SD}, \mathrm{kg} / \mathrm{m}^{2}$ & $24.5 \pm 3.7$ & $24.5 \pm 2.6$ & 0.983 \\
\hline Tumor location, n (\%) & & & 0.860 \\
\hline AEG & $22(56.3 \%)$ & $15(60 \%)$ & \\
\hline Upper third of the stomach & $17(43.6 \%)$ & $10(40 \%)$ & \\
\hline Proximal margin, median (IQR), cm & $2.0(1.5-2.0)$ & $2.0(1.2-3.0)$ & 0.408 \\
\hline Distal margin, median (IQR), cm & $5.0(2.5-6.0)$ & $5.0(3.0-7.5)$ & 0.293 \\
\hline Differentiation, $\mathrm{n}(\%)$ & & & 0.887 \\
\hline Well & $6(15.4 \%)$ & $5(20.0 \%)$ & \\
\hline Moderate & $17(43.6 \%)$ & $10(40.0 \%)$ & \\
\hline Poor & $16(41.0 \%)$ & $10(40.0 \%)$ & \\
\hline Lauren classification, $\mathrm{n}(\%)$ & & & 0.788 \\
\hline Intestinal & $24(61.5 \%)$ & $14(56.0 \%)$ & \\
\hline Diffuse & $7(18.0 \%)$ & $4(16.0 \%)$ & \\
\hline Mixed & $8(20.5 \%)$ & $7(28.0 \%)$ & \\
\hline Tumor size, median (IQR), cm & $3.0(2.0-4.0)$ & $1.5(1.2-3.0)$ & 0.010 \\
\hline pT stage ${ }^{a}, n(\%)$ & & & 0.033 \\
\hline T1a & $8(20.5 \%)$ & $5(20.0 \%)$ & \\
\hline T1b & $9(23.1 \%)$ & $13(52.0 \%)$ & \\
\hline
\end{tabular}

EG, esophagogastrostomy; DT, double tract reconstruction; SD, standard deviation; BMI, body mass index; AEG, adenocarcinoma of the esophagogastric junction; IQR, interquartile range; LNs, lymph nodes; LND, lymph node dissection; LVI, lymphovascular invasion; PNI, perineural invasion.

a 8th UICC/AJCC TNM staging system for gastric cancer

Statistically significant values are in bold. 


\begin{tabular}{|c|c|c|c|}
\hline Clinicopathological characteristics & EG $(n=39)$ & DT $(n=25)$ & $P$ value \\
\hline T2 & $8(20.5 \%)$ & $6(24.0 \%)$ & \\
\hline T3 & $10(25.6 \%)$ & $1(4.0 \%)$ & \\
\hline $\mathrm{T} 4 \mathrm{a}$ & $4(10.3 \%)$ & $0(0.0 \%)$ & \\
\hline $\mathrm{T} 4 \mathrm{~b}$ & $0(0.0 \%)$ & $0(0.0 \%)$ & \\
\hline pN stage ${ }^{a}, \mathrm{n}(\%)$ & & & 0.034 \\
\hline NO & $24(61.5 \%)$ & $23(92.0 \%)$ & \\
\hline N1 & $5(12.8 \%)$ & $2(8.0 \%)$ & \\
\hline N2 & $8(20.5 \%)$ & $0(0.0 \%)$ & \\
\hline N3a & $2(5.2 \%)$ & $0(0.0 \%)$ & \\
\hline N3b & $0(0.0 \%)$ & $0(0.0 \%)$ & \\
\hline pTNM stage ${ }^{\mathrm{a}}, \mathrm{n}(\%)$ & & & 0.031 \\
\hline IA & $16(41.0 \%)$ & $17(68.0 \%)$ & \\
\hline IB & $5(12.8 \%)$ & $6(24.0 \%)$ & \\
\hline$\| A$ & $4(10.3 \%)$ & $2(8.0 \%)$ & \\
\hline IIB & $8(20.5 \%)$ & $0(0.0 \%)$ & \\
\hline IIIA & $4(10.3 \%)$ & $0(0.0 \%)$ & \\
\hline IIIB & $2(5.1 \%)$ & $0(0.0 \%)$ & \\
\hline IIIIC & $0(0.0 \%)$ & $0(0.0 \%)$ & \\
\hline Number of harvested LNs, median (IQR) & $22.5(17.0-31.0)$ & $19.0(16.3-23.0)$ & 0.055 \\
\hline Degree of LND, $n(\%)$ & & & 0.045 \\
\hline D1 & $8(20.5 \%)$ & $11(44.0 \%)$ & \\
\hline D10 & $31(79.5 \%)$ & $14(56.0 \%)$ & \\
\hline LVI, n (\%) & & & 0.080 \\
\hline
\end{tabular}

EG, esophagogastrostomy; DT, double tract reconstruction; SD, standard deviation; BMI, body mass index; AEG, adenocarcinoma of the esophagogastric junction; IQR, interquartile range; LNs, lymph nodes; LND, lymph node dissection; LVI, lymphovascular invasion; PNI, perineural invasion.

a 8th UICC/AJCC TNM staging system for gastric cancer

Statistically significant values are in bold. 


\begin{tabular}{|c|c|c|c|}
\hline Clinicopathological characteristics & EG $(n=39)$ & DT $(n=25)$ & $P$ value \\
\hline No & $23(59.0 \%)$ & $20(80.0 \%)$ & \\
\hline Yes & $16(41.0 \%)$ & $5(20.0 \%)$ & \\
\hline PNI, n (\%) & & & 0.044 \\
\hline No & $24(61.5 \%)$ & $22(88.0 \%)$ & \\
\hline Yes & $15(38.5 \%)$ & $3(12.0 \%)$ & \\
\hline Adjuvant chemotherapy, n (\%) & & & 0.035 \\
\hline No & $16(41.0 \%)$ & $17(68.0 \%)$ & \\
\hline Yes & $23(59.0 \%)$ & $8(32.0 \%)$ & \\
\hline \multicolumn{4}{|c|}{$\begin{array}{l}\text { EG, esophagogastrostomy; DT, double tract reconstruction; SD, standard deviation; BMI, body mass } \\
\text { index; AEG, adenocarcinoma of the esophagogastric junction; IQR, interquartile range; LNs, lymph } \\
\text { nodes; LND, lymph node dissection; LVI, lymphovascular invasion; PNI, perineural invasion. }\end{array}$} \\
\hline \multicolumn{4}{|c|}{ a 8th UICC/AJCC TNM staging system for gastric cancer } \\
\hline Statistically significant values are it & & & \\
\hline
\end{tabular}

\section{Perioperative parameters and survival}

The two groups were well balanced in terms of length of postoperative hospital stay, blood loss volume, and postoperative complications (Table 2). The operation duration in the double tract reconstruction group was longer than that in the esophagogastrostomy group ( $240 \mathrm{~min}$ vs $195 \mathrm{~min}, P=0.001$ ). The $30-$ day reoperation rate and 30-day mortality rate were comparable between the two groups. 
Table 2

Intraoperative and postoperative parameters of the patients

\begin{tabular}{|llll|}
\hline Perioperative parameters & EG $(\mathbf{n}=39)$ & DT $(\mathbf{n = 2 5})$ & Pvalue \\
\hline Postoperative hospital stay, median (IQR), day & $11(10-14)$ & $13(11-18)$ & 0.220 \\
\hline Operation duration, median (IQR), min & $195(170-240)$ & $240(210-270)$ & 0.001 \\
\hline Blood loss volume, median (IQR), ml & $100(50-100)$ & $50(50-100)$ & 0.189 \\
\hline Postoperative complication*, $\mathrm{n}(\%)$ & & & 1.000 \\
\hline No & $32(82.1 \%)$ & $21(84.0 \%)$ & \\
\hline Yes & $7(17.9 \%)$ & $4(16.0 \%)$ & \\
\hline Anastomotic leakage & $4(10.3 \%)$ & $2(8.0 \%)$ & \\
\hline Anastomotic bleeding & $0(0.0 \%)$ & $0(0.0 \%)$ & \\
\hline Anastomotic stenosis & $0(0.0 \%)$ & $0(0.0 \%)$ & \\
\hline Effusion & $3(7.7 \%)$ & $2(8.0 \%)$ & \\
\hline Infection & $4(10.3 \%)$ & $2(8.0 \%)$ & \\
\hline Gastrointestinal dysfunction & $2(5.1 \%)$ & $0(0.0 \%)$ & \\
\hline 30-day reoperation rate & $0(0.0 \%)$ & $0(0.0 \%)$ & \\
\hline 30-day mortality rate & $0(0.0 \%)$ & $0(0.0 \%)$ & \\
\hline EG, esophagogastrostomy; DT, double tract reconstruction; IQR, interquartile range. & \\
\hline * Complications were defined according to the Clavien-Dindo classification system. & \\
\hline Statistically significant values are in bold. & & & \\
\hline
\end{tabular}

As of May 2020, the median follow-up time was 23.1 months (range: 11.4-63.2 months). The 5-year DFS rates in the esophagogastrostomy group and double tract reconstruction group were $90.9 \%$ and $88.9 \%$, respectively $(P=0.707$, Fig. 1$)$.

\section{Reflux esophagitis}

Reflux esophagitis occurred in twelve patients (30.8\%) in the esophagogastrostomy group and two patients $(8.0 \%)$ in the double tract reconstruction group $(P=0.032$, Table 3$)$. Of the twelve patients who were diagnosed with reflux esophagitis in the esophagogastrostomy group, nine patients were classified as level $A$ and three patients were classified as level B according to the Los Angeles classification. In the double tract reconstruction group, both patients were classified as level A according to the Los Angeles classification. 
Table 3

Evaluation of postoperative reflux esophagitis of the patients

\begin{tabular}{|lcc|}
\hline \multicolumn{4}{|c|}{ EG $(\mathbf{n = 3 9 )}$} & DT $(\mathbf{n = 2 5 )}$ & Pvalue \\
\hline Reflux esophagitis & & 0.032 \\
No & $27(69.2 \%)$ & $23(92.0 \%)$ \\
Yes & $12(30.8 \%)$ & $2(8.0 \%)$ \\
\hline Los Angeles classification & & \\
\hline A & $9(23.1 \%)$ & $2(8.0 \%)$ \\
\hline B & $3(7.7 \%)$ & $0(0.0 \%)$ \\
\hline EG, esophagogastrostomy; DT, double tract reconstruction. \\
\hline Statistically significant values are in bold. \\
\hline
\end{tabular}

To identify the risk factors for postoperative reflux esophagitis, a univariate analysis was performed (Table 4). Then, all factors with a $P$ value less than 0.2 were included in the multivariable logistic regression analysis. Finally, the reconstruction method was found to be the only independent risk factor for reflux esophagitis $(P=0.004$, Table 4$)$. 
Table 4

Univariate analysis and multivariate logistic regression analysis of reflux esophagitis

\begin{tabular}{|c|c|c|c|c|}
\hline Univariate analysis & $P$ value & Multivariate analysis & OR $(95 \% \mathrm{Cl})$ & $P$ value \\
\hline Age & 0.696 & Reconstruction method & & 0.004 \\
\hline Gender & 0.644 & EG & 1 & \\
\hline BMI & 0.408 & DT & $0.078(0.009$ & 674) \\
\hline Tumor location & 1.000 & & & \\
\hline Proximal margin & 0.568 & & & \\
\hline Distal margin & 0.418 & & & \\
\hline Differentiation & 1.000 & & & \\
\hline Lauren classification & 0.143 & & & \\
\hline Tumor size & 0.689 & & & \\
\hline pT stage & 0.153 & & & \\
\hline pN stage & 0.518 & & & \\
\hline pTNM stage & 0.405 & & & \\
\hline Number of harvested LNs & 0.179 & & & \\
\hline Degree of LND & 0.526 & & & \\
\hline LVI & 1.000 & & & \\
\hline PNI & 0.085 & & & \\
\hline Adjuvant chemotherapy & 0.232 & & & \\
\hline Operation duration & 0.128 & & & \\
\hline Blood loss volume & 0.113 & & & \\
\hline Postoperative hospital stay & 0.318 & & & \\
\hline Postoperative complication & 1.000 & & & \\
\hline Reconstruction method & 0.032 & & & \\
\hline \multicolumn{5}{|c|}{$\begin{array}{l}\text { EG, esophagogastrostomy; DT, double tract reconstruction; BMI, body mass index; LNs, lymph nodes; } \\
\text { LND, lymph node dissection; LVI, lymphovascular invasion; PNI, perineural invasion; OR, odds ratio; Cl, } \\
\text { confidence interval. }\end{array}$} \\
\hline \multicolumn{5}{|c|}{ Statistically significant values are in bold. } \\
\hline
\end{tabular}

\section{Quality of life}


The scores of the EORTC QLQ-C30 version 3.0 questionnaire were compared between the esophagogastrostomy and double tract reconstruction groups (Table 5, Fig. 2, and Fig. 3). The global health status score was higher in the double tract reconstruction group than in the esophagogastrostomy group (83.3 vs. 50.0, $P<0.001)$. Compared with patients in the esophagogastrostomy group, patients in the double tract reconstruction group had better emotional functioning $(P<0.001)$, and complained less about nausea and vomiting $(P<0.001)$, pain $(P=0.039)$, insomnia $(P=0.003)$, and appetite loss $(P<$ 0.001). The results of the EORTC QLQ-STO22 questionnaire were also compared between groups (Table 6 and Fig. 4). Patients in the double tract reconstruction group complained less about dysphagia $(P=$ $0.030)$, pain $(P=0.008)$, reflux $(P<0.001)$, eating $(P<0.001)$, anxiety $(P<0.001)$, dry mouth $(P=0.007)$, and taste $(P=0.001)$ than those in the esophagogastrostomy group.

Table 5

The scores of the EORTC QLQ-C30 version 3.0 questionnaire

\begin{tabular}{|llll|}
\hline & EG, median (IQR) & DT, median (IQR) & Pvalue \\
\hline Global health status & $50.0(33.3-50.0)$ & $83.3(66.7-83.3)$ & $<0.001$ \\
\hline Functional scales & & & \\
\hline Physical functioning & $80.0(66.7-86.7)$ & $80(66.7-86.7)$ & 0.600 \\
\hline Role functioning & $66.7(66.7-66.7)$ & $66.7(66.7-83.3)$ & 0.108 \\
\hline Emotional functioning & $50.0(41.7-66.7)$ & $66.7(66.7-79.2)$ & $<0.001$ \\
\hline Cognitive functioning & $100.0(66.7-100.0)$ & $100.0(75.0-100.0)$ & 0.142 \\
\hline Social functioning & $66.7(66.7-66.7)$ & $66.7(66.7-83.3)$ & 0.073 \\
\hline Symptom scales & & & \\
\hline Fatigue & $33.3(33.3-33.3)$ & $33.3(0.0-33.3)$ & 0.473 \\
\hline Nausea and vomiting & $50.0(50.0-83.3)$ & $16.7(16.7-16.7)$ & $<0.001$ \\
\hline Pain & $16.7(16.7-33.3)$ & $16.7(0.0-33.3)$ & 0.039 \\
\hline Dyspnea & $0.0(0.0-33.3)$ & $0.0(0.0-0.0)$ & 0.072 \\
\hline Insomnia & $33.3(0.0-33.3)$ & $0.0(0.0-0.0)$ & 0.003 \\
\hline Appetite loss & $33.3(33.3-33.3)$ & $0.0(0.0-33.3)$ & $<0.001$ \\
\hline Constipation & $0.0(0.0-33.3)$ & $0.0(0.0-0.0)$ & 0.082 \\
\hline Diarrhea & $0.0(0.0-33.3)$ & $0.0(0.0-0.0)$ & 0.129 \\
\hline Financial difficulties & $33.3(0.0-33.3)$ & $33.3(0.0-33.3)$ & 0.638 \\
\hline EG, esophagogastrostomy; IQR, interquartile range; DT, double tract reconstruction. & \\
\hline Statistically significant values are in bold. & & \\
\hline
\end{tabular}


Table 6

The scores of the EORTC QLQ-STO22 questionnaire

\begin{tabular}{|llll|}
\hline Symptom scales & EG, median (IQR) & DT, median (IQR) & $P$ value \\
\hline Dysphagia & $22.2(11.1-33.3)$ & $11.1(11.1-22.2)$ & 0.030 \\
\hline Pain & $33.3(16.7-41.7)$ & $25.0(12.5-25.0)$ & 0.008 \\
\hline Reflux & $88.9(66.7-100.0)$ & $33.3(0.0-44.4)$ & $<0.001$ \\
\hline Eating & $41.7(33.3-66.7)$ & $25.0(20.8-33.3)$ & $<0.001$ \\
\hline Anxiety & $77.8(66.7-100.0)$ & $55.6(44.4-66.7)$ & $<0.001$ \\
\hline Dry mouth & $33.3(33.3-66.7)$ & $33.3(0.0-33.3)$ & 0.007 \\
\hline Taste & $66.7(33.3-66.7)$ & $33.3(0.0-33.3)$ & 0.001 \\
\hline Body image & $66.7(66.7-100.0)$ & $66.7(66.7-100.0)$ & 0.807 \\
\hline Hair loss & $0.0(0.0-0.0)$ & $0.0(0.0-33.3)$ & 0.494 \\
\hline EG, esophagogastrostomy; IQR, interquartile range; DT, double tract reconstruction. \\
\hline Statistically significant values are in bold. & & \\
\hline
\end{tabular}

In the simple linear regression analysis of global health status, the results showed that pT stage $(P=$ $0.035)$, pN stage $(P=0.016)$, pTNM stage $(P=0.015)$, PNI $(P=0.022)$, operation duration $(P=0.008)$, reflux esophagitis $(P=0.001)$, and reconstruction method $(P=0.001)$ were all correlated with global health status (Table 7). All variables with a $P$ value less than 0.1 were included in the multiple linear regression analysis. A linear regression equation was established, and the results showed a linear relationship between the global health status score and reconstruction method, postoperative complications, reflux esophagitis, and operation duration (Table 7 and Table 8). 
Table 7

Simple and multiple linear regression analyses of global health status

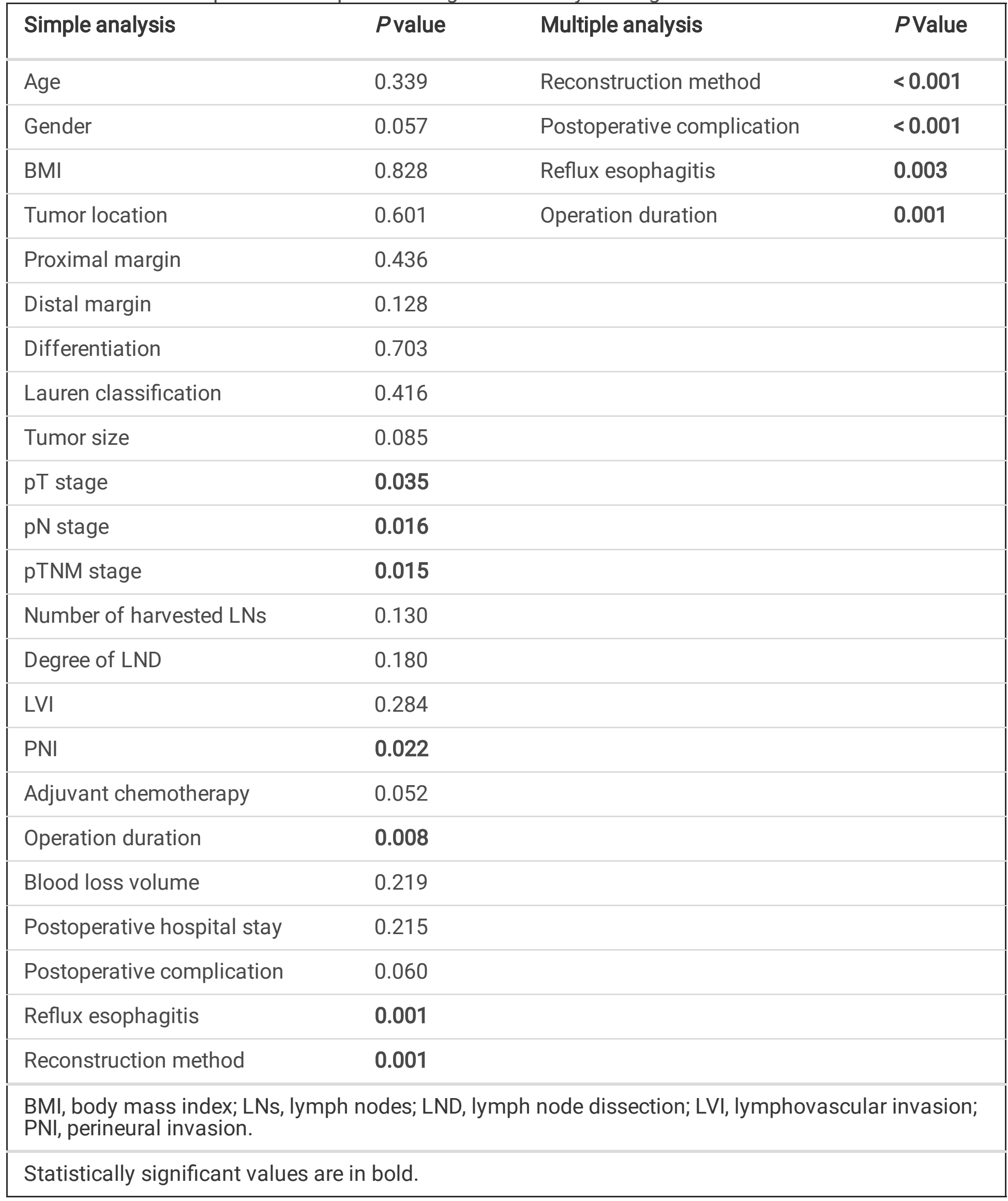


Table 8

Multiple linear regression equation of global health status
Linear regression equation
Adjusted R-squared
0.791
GHS score $=23.8 \cdot \mathrm{RM}+0.1 \cdot \mathrm{OD}-14.3 \cdot \mathrm{PC}-9.6 \cdot \mathrm{RE}+44.2$
RM: double tract reconstruction $=1$, esophagogastrostomy $=0$
OD: operation duration, expressed in minutes
PC: without complication $=0$, with complication $=1$
RE: without reflux esophagitis $=0$, with reflux esophagitis $=1$

F-value

$P$ value

22.961

$<0.001$

GHS, global health status; RM, reconstruction method; PC, postoperative complication; RE, reflux esophagitis; OD, operation duration.

Statistically significant values are in bold.

\section{Discussion}

Esophagogastrostomy reconstruction is a traditional reconstruction method after proximal gastrectomy. However, esophagogastrostomy is associated with a high incidence of postoperative reflux esophagitis. To address this disadvantage, several modified reconstruction methods, including double tract reconstruction, have been attempted.

The safety and feasibility of double tract reconstruction after proximal gastrectomy have been of great concern in recent years. In the present study, the results showed that there was no significant difference in blood loss volume, length of postoperative hospital stay or postoperative complications between esophagogastrostomy and double tract reconstruction. The operation duration was longer in the double tract reconstruction group than in the esophagogastrostomy group in this study. Considering that the double tract reconstruction procedure is more complex than the esophagogastrostomy procedure, it is reasonable that the operation duration of double tract reconstruction was longer than that of esophagogastrostomy. On the other hand, whether double tract reconstruction causes a high rate of complications because of the technical complexity and increased number of anastomoses has been questioned $[10,11,19]$. In the present study, postoperative complications, blood loss volume and length of postoperative hospital stay were all comparable between the two reconstruction methods. These results suggest that the complexity of the surgical procedure dose not increase the risk of the operation.

The anti-reflux function of double tract reconstruction after proximal gastrectomy has been investigated in previous studies $[12,13]$. However, most of the results are based on studies with exceedingly small samples. In the present study, the rates of reflux esophagitis in the esophagogastrostomy group and double tract reconstruction group were $30.8 \%$ and $8.0 \%$, respectively $(P=0.032)$. Moreover, multivariate logistic regression analysis showed that the reconstruction method was the only independent risk factor 
for reflux esophagitis $(P=0.004)$. Double tract reconstruction is a protective factor against postoperative reflux esophagitis after proximal gastrectomy. Considering the reconstruction procedure, it is possible that the mechanism of anti-reflux function is mainly attributed to the lifted jejunum between esophagojejunostomy and gastrojejunostomy [20, 21].

Many studies have reported nutritional status after proximal gastrectomy [13, 22, 23]. However, postoperative quality of life after proximal gastrectomy has rarely been reported before. Thus, the present study compared quality of life after proximal gastrectomy with the EORTC QLQ-C30 version 3.0 and QLQST022 questionnaires between esophagogastrostomy and double tract reconstruction. According to the EORTC scoring manual, a higher score represented a better level of functioning, or a worse level of symptoms [24]. In the present study, double tract reconstruction had obvious advantages over esophagogastrostomy based on the EORTC QLQ-C30 questionnaire, including in global health status, emotional functioning, nausea and vomiting, pain, insomnia, and appetite loss (Table 5). For the EORTC QLQ-ST022 questionnaire, symptoms including dysphagia, pain, reflux, eating, anxiety, dry mouth, and taste were much milder in the double tract reconstruction group than in the esophagogastrostomy group (Table 6). The mechanism of the anti-reflux function of double tract reconstruction has been discussed in the previous paragraph of this section. Furthermore, previous studies reported that the prevention of reflux esophagitis might improve the psychological, physical, and social functioning of patients [25]. Moreover, the prevention of reflux esophagitis could also relieve symptoms such as nausea, vomiting, pain, insomnia, appetite loss, dysphagia, eating, anxiety, and dry mouth [26-31]. Therefore, the better scores in the double tract reconstruction group might be due to the superiority of the surgical procedure itself in preventing reflux esophagitis after proximal gastrectomy.

Considering that the global health status score in the EORTC QLQ-C30 questionnaire can reflect overall quality of life, a linear regression analysis was performed to investigate the related factors [24]. The results showed that reconstruction method $(P<0.001)$, postoperative complications $(P<0.001)$, reflux esophagitis $(P=0.003)$, and operation duration $(P=0.001)$ had a linear relationship with the global health status score (Table 8). The mechanism of double tract reconstruction in preventing reflux esophagitis and improving quality of life has been discussed in the previous paragraph. Furthermore, some studies have reported that postoperative complications could induce physical and mental discomfort and impair quality of life [32, 33]. Interestingly, operation duration was also identified in the equation. The operation duration could reflect how elaborate the operation was. Theoretically, an elaborate operation could protect the nerves better, and better preserve organ function [34-36]. Therefore, a longer operation duration might improve quality of life after the operation.

The present study also has some limitations. First, selection bias was difficult to avoid because this was a retrospective study. The pathological stage was not comparable between the two groups, resulting in differences in tumor size, degree of LND, PNI, and adjuvant chemotherapy. This phenomenon was mainly caused by the different choices of the patients. In our hospital, the reconstruction method was discussed and determined by both the patients and surgeons. Patients with early-stage disease were more likely to choose double tract reconstruction after learning about its superiority because these patients might pay 
more attention to quality of life after the operation. Thus, the shared decision-making method might be the cause of the bias. However, multivariable logistic regression analysis and multiple linear regression analysis could neutralize the confounding effects of these factors. Second, the sample size of the present study was not large enough, which might make the results of this study less convincing. Further largescale, prospective, randomized controlled trial is needed to validate the results of the study.

\section{Conclusions}

Double tract reconstruction after proximal gastrectomy was comparable with esophagogastrostomy in terms of perioperative safety and 5-year DFS. Double tract reconstruction could better prevent reflux esophagitis and improve quality of life. The reconstruction method was the only independent risk factor for reflux esophagitis. There was a linear relationship between global health status score and reconstruction method, reflux esophagitis, postoperative complications, and operation duration.

\section{Abbreviations}

DFS, disease-free survival; EORTC, European Organization for Research and Treatment of Cancer; UICC, Union for International Cancer Control; AJCC, American Joint Committee on Cancer; LND, lymph node dissection; BMI, body mass index; LNs, lymph nodes; LVI, lymphovascular invasion; PNI, perineural invasion.

\section{Declarations}

\section{Ethics approval and consent to participate}

This study was conducted in accordance with the Declaration of Helsinki and had been authorized by the Ethics Committee of Peking University Cancer Hospital (Reference No. 2018KT97) before beginning the study. Each patient in this study provided signed informed consent.

\section{Consent for publication}

Not applicable

\section{Availability of data and materials}

The datasets used and analyzed during the current study are available from the corresponding author on reasonable request.

\section{Competing interests}


The authors declare that they have no competing interests

\section{Funding}

This study was supported by the Research Incubation Project, Beijing Municipal Administration of Hospitals (PX2019039). The funder was not involved in the design of the study and collection, analysis, and interpretation of data and in writing the manuscript.

\section{Authors' contributions:}

J-FJ and Z-DB designed this study and were in charge of its coordination. XJ, C-GJ, KJ, JZ, X-JW, X-LZ, Z$\mathrm{YJ}, \mathrm{TF}, \mathrm{BF}$ and $\mathrm{A}-\mathrm{QW}$ participated in the data collection, statistical analysis, and the interpretation of data and results. XJ, C-GJ and KJ drafted the manuscript. XJ, C-GJ and KJ are joint first authors. All authors have read and approved the final manuscript.

\section{Acknowledgments}

The authors were grateful for the staff members at the database center of the Peking University Cancer Hospital and Institute for assistance in data collection.

\section{References}

1. Bray F, Ferlay J, Soerjomataram I, Siegel RL, Torre LA, Jemal A. Global cancer statistics 2018 : GLOBOCAN estimates of incidence and mortality worldwide for 36 cancers in 185 countries. CA Cancer J Clin. 2018;68(6):394-424.

2. Wang A, Li Z, Wang Q, Bai Y, Ji X, Fu T, et al. Diagnostic value of negative enrichment and immune fluorescence in situ hybridization for intraperitoneal free cancer cells of gastric cancer. Chin $\mathrm{J}$ Cancer Res. 2019;31(6):945-54.

3. Sun W, Deng J, Zhang N, Liu H, Liu J, Gu P, et al. Prognostic impact of D2-plus lymphadenectomy and optimal extent of lymphadenectomy in advanced gastric antral carcinoma: Propensity score matching analysis. Chin J Cancer Res. 2020;32(1):51-61.

4. Haruta S, Shinohara H, Hosogi H, Ohkura Y, Kobayashi N, Mizuno A, et al. Proximal gastrectomy with exclusion of no. 3b lesser curvature lymph node dissection could be indicated for patients with advanced upper-third gastric cancer. Gastric Cancer. 2017;20(3):528-35.

5. 10.1007/s10120-020-01042-y

Japanese Gastric Cancer A. Japanese gastric cancer treatment guidelines 2018 (5th edition). Gastric Cancer. 2020; doi:10.1007/s10120-020-01042-y. 
6. Hiki N, Nunobe S, Kubota T, Jiang X. Function-preserving gastrectomy for early gastric cancer. Ann Surg Oncol. 2013;20(8):2683-92.

7. Takayama T, Matsumoto S, Wakatsuki K, Tanaka T, Migita K, Ito M, et al. Novel laparoscopic procedure for treating proximal early gastric cancer: laparoscopy-assisted pylorus-preserving nearly total gastrectomy. Surg Today. 2014;44(12):2332-8.

8. Koeda K, Nishizuka S, Wakabayashi G. Minimally invasive surgery for gastric cancer: the future standard of care. World J Surg. 2011;35(7):1469-77.

9. Kumagai K, Shimizu K, Yokoyama N, Aida S, Arima S, Aikou T, et al. Questionnaire survey regarding the current status and controversial issues concerning reconstruction after gastrectomy in Japan. Surg Today. 2012;42(5):411-8.

10. Ichikawa D, Ueshima Y, Shirono K, Kan K, Shioaki Y, Lee CJ, et al. Esophagogastrostomy reconstruction after limited proximal gastrectomy. Hepatogastroenterology. 2001;48(42):1797-801.

11. Nakamura M, Nakamori M, Ojima T, Katsuda M, lida T, Hayata $K$, et al. Reconstruction after proximal gastrectomy for early gastric cancer in the upper third of the stomach: an analysis of our 13-year experience. Surgery. 2014;156(1):57-63.

12. Wang $S$, Lin S, Wang H, Yang J, Yu P, Zhao Q, et al. Reconstruction methods after radical proximal gastrectomy: A systematic review. Med (Baltim). 2018;97(11):e0121.

13. Ahn SH, Jung DH, Son SY, Lee CM, Park DJ, Kim HH. Laparoscopic double-tract proximal gastrectomy for proximal early gastric cancer. Gastric Cancer. 2014;17(3):562-70.

14. Japanese Gastric Cancer A. Japanese classification of gastric carcinoma: 3rd English edition. Gastric Cancer. 2011;14(2):101-12.

15. Ji X, Bu ZD, Yan Y, Li ZY, Wu AW, Zhang LH, et al. The 8th edition of the American Joint Committee on Cancer tumor-node-metastasis staging system for gastric cancer is superior to the 7th edition: results from a Chinese mono-institutional study of 1663 patients. Gastric Cancer. 2018;21(4):643-52.

16. Japanese Gastric Cancer A. Japanese gastric cancer treatment guidelines 2014 (ver. 4). Gastric Cancer. 2017;20(1):1-19.

17. Li Z, Bai B, Ji G, Li J, Zhao Q. Relationship between Clavien-Dindo classification and long-term survival outcomes after curative resection for gastric cancer: A propensity score-matched analysis. Int J Surg. 2018;60:67-73.

18. Sugiura T, Iwakiri K, Kotoyori M, Kobayashi M. Relationship between severity of reflux esophagitis according to the Los Angeles classification and esophageal motility. J Gastroenterol. 2001;36(4):226-30.

19. Seshimo A, Miyake K, Amano K, Aratake K, Kameoka S. Clinical outcome of esophagogastrostomy after proximal gastrectomy for gastric cancer. Hepatogastroenterology. 2013;60(123):616-9.

20. Jung DH, Lee Y, Kim DW, Park YS, Ahn SH, Park DJ, et al. Laparoscopic proximal gastrectomy with double tract reconstruction is superior to laparoscopic total gastrectomy for proximal early gastric cancer. Surg Endosc. 2017;31(10):3961-69. 
21. Wang L, Xia Y, Jiang T, Li F, Wang W, Zhang D, et al. Short-Term Surgical Outcomes of Laparoscopic Proximal Gastrectomy With Double-Tract Reconstruction Versus Laparoscopic Total Gastrectomy for Adenocarcinoma of Esophagogastric Junction: A Matched-Cohort Study. J Surg Res. 2020;246:29299.

22. Nakamura $\mathrm{M}$, Yamaue $\mathrm{H}$. Reconstruction after proximal gastrectomy for gastric cancer in the upper third of the stomach: a review of the literature published from 2000 to 2014. Surg Today. 2016;46(5):517-27.

23. Nomura E, Lee SW, Kawai M, Yamazaki M, Nabeshima K, Nakamura K, et al. Functional outcomes by reconstruction technique following laparoscopic proximal gastrectomy for gastric cancer: double tract versus jejunal interposition. World J Surg Oncol. 2014;12:20.

24. Fayers P, Aaronson N, Bjordal K, Groenvold M, Curran D, Bottomley A. The EORTC QLQ-C30 Scoring Manual (3rd Edition). European Organisation for Research and Treatment of Cancer, Brussels. 2001.

25. Hongo M, Kinoshita Y, Miwa H, Ashida K. The demographic characteristics and health-related quality of life in a large cohort of reflux esophagitis patients in Japan with reference to the effect of lansoprazole: the REQUEST study. J Gastroenterol. 2008;43(12):920-7.

26. Javadi S, Shafikhani AA. Anxiety and depression in patients with gastroesophageal reflux disorder. Electron Physician. 2017;9(8):5107-12.

27. Battaglia E, Grassini M, Navino M, Niola P, Verna C, Mazzocchi A, et al. Water load test before and after PPI therapy in patients with gastro-oesophageal reflux disease. Dig Liver Dis. 2007;39(12):1052-6.

28. Dimenas E, Glise H, Hallerback B, Hernqvist H, Svedlund J, Wiklund I. Quality of life in patients with upper gastrointestinal symptoms. An improved evaluation of treatment regimens? Scand $\mathrm{J}$ Gastroenterol. 1993;28(8):681-7.

29. Oh JH. Atypical symptoms are related to typical symptoms rather than histologic and endoscopic esophagitis. J Neurogastroenterol Motil. 2012;18(3):233-5.

30. Yoshikawa H, Furuta K, Ueno M, Egawa M, Yoshino A, Kondo S, et al. Oral symptoms including dental erosion in gastroesophageal reflux disease are associated with decreased salivary flow volume and swallowing function. J Gastroenterol. 2012;47(4):412-20.

31. Shibata T, Nakamura M, Omori T, Tahara T, Ichikawa Y, Okubo M, et al. Association between individual response to food taste and gastroesophageal symptoms. J Dig Dis. 2015;16(6):337-41.

32. Kauppila JH, Ringborg C, Johar A, Lagergren J, Lagergren P. Health-related quality of life after gastrectomy, esophagectomy, and combined esophagogastrectomy for gastroesophageal junction adenocarcinoma. Gastric Cancer. 2018;21(3):533-41.

33. Diaz De Liano A, Oteiza Martinez F, Ciga MA, Aizcorbe M, Cobo F, Trujillo R. Impact of surgical procedure for gastric cancer on quality of life. Br J Surg. 2003;90(1):91-4.

34. Kim SM, Cho J, Kang D, Oh SJ, Kim AR, Sohn TS, et al. A Randomized Controlled Trial of Vagus Nerve-preserving Distal Gastrectomy Versus Conventional Distal Gastrectomy for Postoperative Quality of Life in Early Stage Gastric Cancer Patients. Ann Surg. 2016;263(6):1079-84. 
35. Inokuchi M, Sugita H, Otsuki S, Sato Y, Nakagawa M, Kojima K. Long-term effectiveness of preserved celiac branch of vagal nerve after Roux-en-Y reconstruction in laparoscopy-assisted distal gastrectomy. Dig Surg. 2014;31(4-5):341-6.

36. Tomita R. A novel surgical procedure of vagal nerve, lower esophageal sphincter, and pyloric sphincter-preserving nearly total gastrectomy reconstructed by single jejunal interposition, and postoperative quality of life. Hepatogastroenterology. 2005;52(66):1895-901.

\section{Figures}

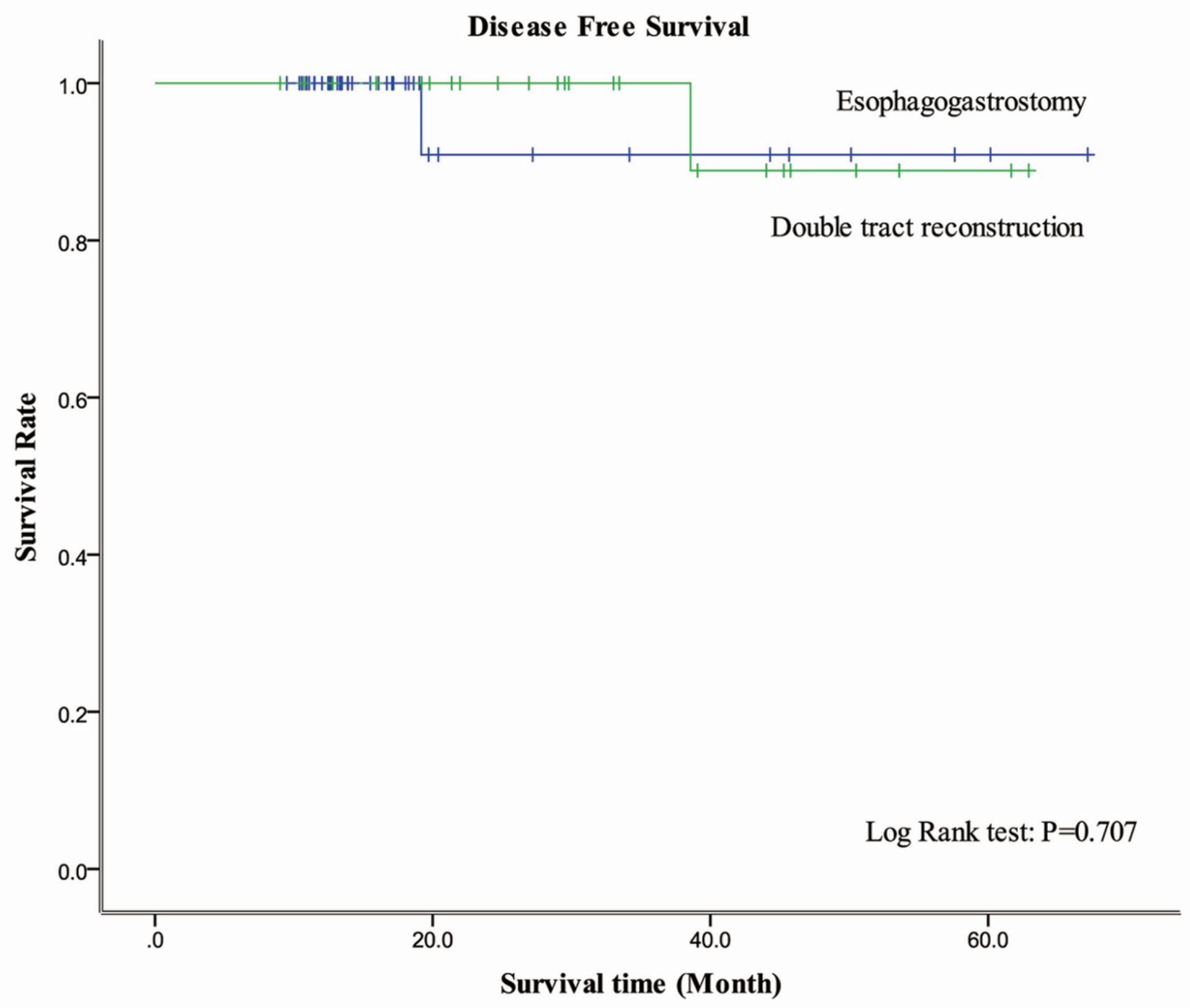

Figure 1 
Five-year DFS curves of patients in the esophagogastrostomy and double tract reconstruction groups. The 5-year DFS rates were comparable between the two groups. The 5-year DFS rates of the esophagogastrostomy and double tract reconstruction groups were $90.9 \%$ and $88.9 \%$, respectively $(\mathrm{P}=$ 0.707).

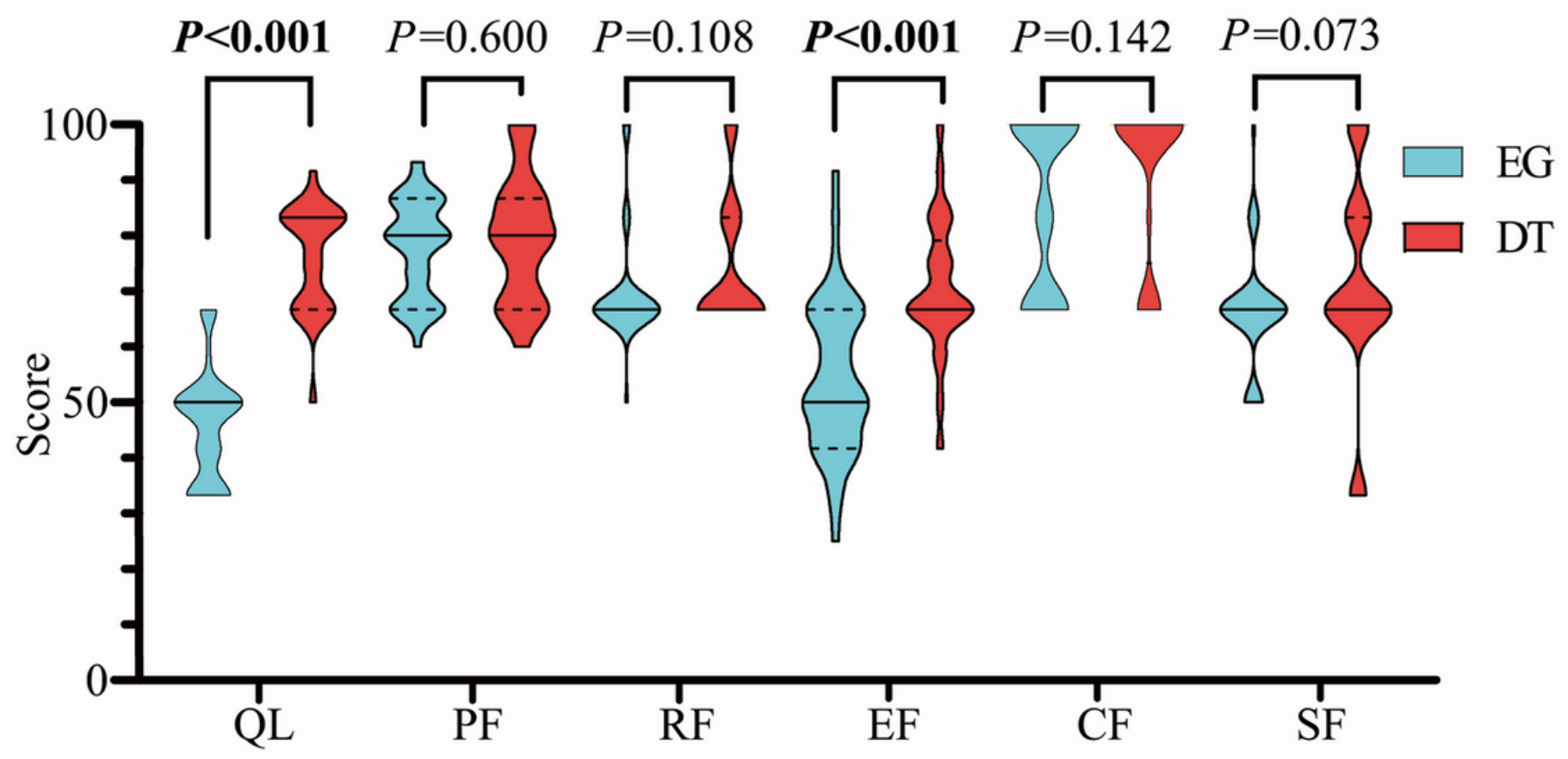

\section{Figure 2}

Violin plots of global health status and functional scales of the EORTC QLQ-C30 questionnaire. Solid lines represent medians and dotted lines represent quartiles. A high score for global health status represented a high quality of life. A high score for a functional scale represented a healthy level of functioning. The patients in the double tract reconstruction group had better global health status $(\mathrm{P}<$ $0.001)$ and emotional functioning $(P<0.001)$ than those in the esophagogastrostomy group. EG, esophagogastrostomy; DT, double tract reconstruction; QL, global health status; PF, physical functioning; $\mathrm{RF}$, role functioning; $\mathrm{EF}$, emotional functioning; $\mathrm{CF}$, cognitive functioning; $\mathrm{SF}$, social functioning. 


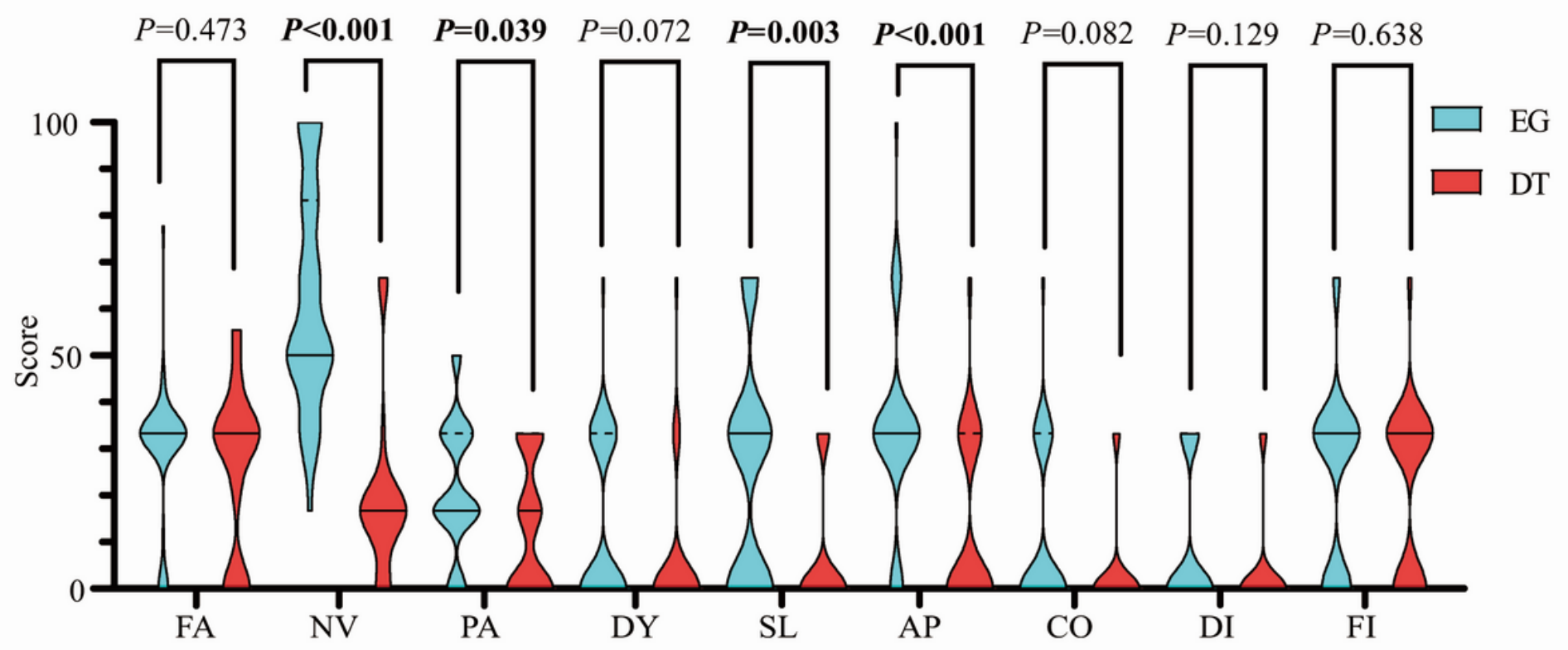

Figure 3

Violin plots of global health status and functional scales of the EORTC QLQ-C30 questionnaire. Solid lines represent medians and dotted lines represent quartiles. A high score for global health status represented a high quality of life. A high score for a functional scale represented a healthy level of functioning. The patients in the double tract reconstruction group had better global health status $(\mathrm{P}<$ $0.001)$ and emotional functioning $(P<0.001)$ than those in the esophagogastrostomy group. EG, esophagogastrostomy; DT, double tract reconstruction; QL, global health status; PF, physical functioning; $\mathrm{RF}$, role functioning; $\mathrm{EF}$, emotional functioning; $C F$, cognitive functioning; $S F$, social functioning. $E G$, esophagogastrostomy; DT, double tract reconstruction; FA, fatigue; NV, nausea and vomiting; PA, pain; DY, dyspnea; SL, insomnia; AP, appetite loss; CO, constipation; DI, diarrhea; FI, financial difficulties. 


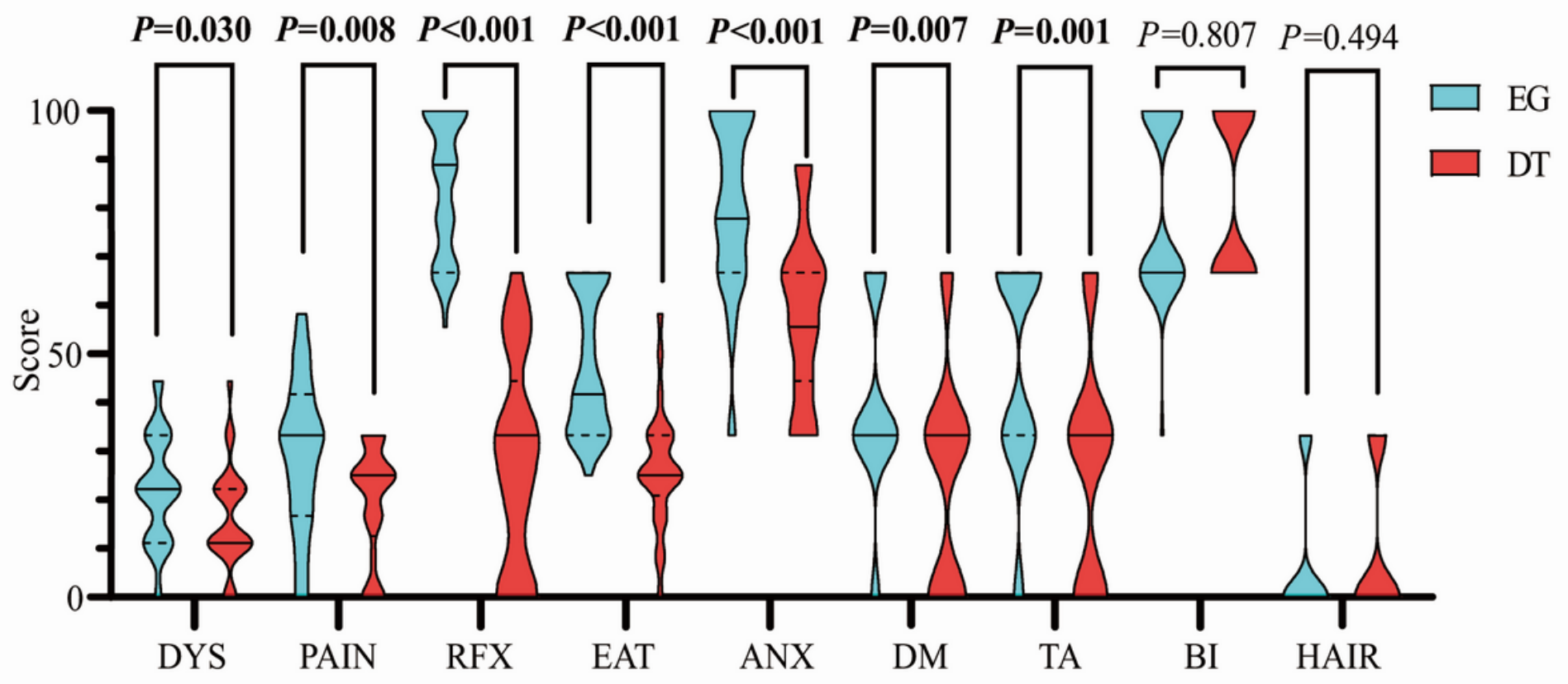

Figure 4

Violin plots of the symptom scales of the EORTC QLQ-STO22 questionnaire. Solid lines represent medians and dotted lines represent quartiles. A higher score represented worse symptoms. Patients in the double tract reconstruction group complained less about dysphagia $(P=0.030)$, pain $(P=0.008)$, reflux $(P<0.001)$, eating $(P<0.001)$, anxiety $(P<0.001)$, dry mouth $(P=0.007)$, and taste $(P=0.001)$ than those in the esophagogastrostomy group. EG, esophagogastrostomy; DT, double tract; DYS, dysphagia; PAIN, pain; RFX, reflux; EAT; eating; ANX, anxiety; DM, dry mouth; TA, taste; BI, body image; HAIR, hair loss. 